\title{
Recognizing A Low-Grade Oncocytic Renal Tumor
}

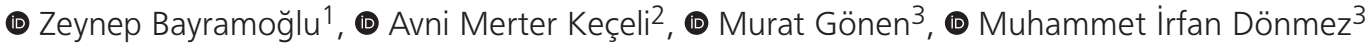 \\ ${ }^{1}$ Konya Training and Research Hospital, Clinic of Pathology, Konya, Turkey \\ 2Konya Training and Research Hospital, Clinic of Radiology, Konya, Turkey \\ ${ }^{3}$ Konya Training and Research Hospital, Clinic of Urology, Konya, Turkey
}

\begin{abstract}

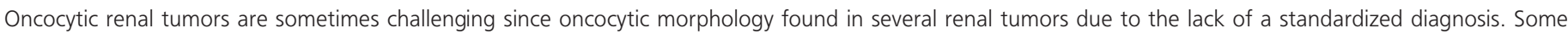

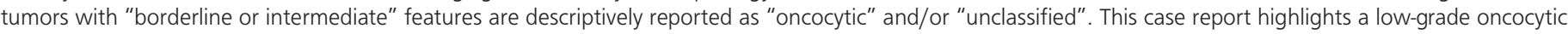
renal tumor.

Keywords: Chromophobe, renal cell carcinoma, hybrid tumor, low-grade, oncocytic tumor
\end{abstract}

\section{Introduction}

Oncocytic renal tumor diagnosis is sometimes challenging due to the oncocytic morphology in several renal tumors. The most common problem is the distinction between eosinophilic variant of chromophobe renal cell carcinoma (ChrRCC) and oncocytoma. Tumors with "borderline or intermediate" features are often descriptively reported as "oncocytic" and/or "unclassified" as main descriptive diagnosis terms preferring one or the other (1). However, some oncocytic tumors still do not fit into any available "oncocytic" tumor categories $(2,3)$. Therefore, a comprehensive morphology, immunohistochemistry, and genetic profile research to precisely define and classify tumors with oncocytic morphology is important (2). This case report highlights a low-grade oncocytic renal tumor.

\section{Case Report}

All procedures performed in this study involving human participant were in accordance with the ethical standards of the institutional and/or national research committee and with the 1964 Helsinki declaration and its later amendments or comparable ethical standards. Consent was obtained from the patient.

A 73-year-old female patient was admitted to the department of pulmonology due to extreme coughing. She had chronic renal failure and hypertension for 10 years. The computed tomography of the chest detected a solid mass in the lower pole of the right kidney (Figure 1). Irregularity was observed in its contour compatible with fibrotic sequela in the middle pole of the ipsilateral kidney. The left kidney was atrophic. A dynamic enhanced magnetic resonance imaging evaluated the renal mass since the patient had compromised renal functions. The right renal mass was well limited and separated from the normal kidney parenchyma. It was overflowing from the kidney contour, but not exceeding the kidney capsule. A pseudocapsule appearance was observed around the mass. The internal lesion

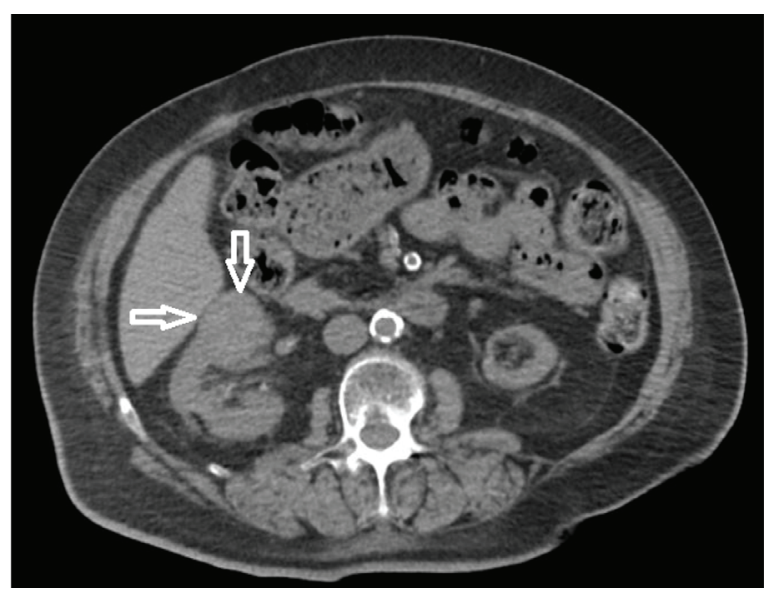

Figure 1. Non-enhanced axial plan CT image showing the right kidney mass (arrows). The mass is well-circumscribed with similar density to the kidney parenchyma. The left kidney is atrophic in size

CT: Computer tomography

Cite this article as: Bayramoğlu Z, Keçeli AM, Gönen M, Dönmez Mi. Recognizing A Low-Grade Oncocytic Renal Tumor. Bull Urooncol 2021;20(4):273-275

Address for Correspondence: Muhammet İrfan Dönmez, Konya Training and Research Hospital, Clinic of Urology, Konya, Turkey Phone: +90 3123051969 E-mail: m irfan83@yahoo.com ORCID-ID: orcid.org/0000-0002-2828-7942 Received: 10.08.2020 Accepted: 16.08.2020 
structure was close to the kidney tissue in $\mathrm{T} 1$ and $\mathrm{T} 2$ weighted images, without significant fat contents. The enhanced image series enhanced the early phase, without contrast washout in the late phase. Pathological lymph node, solid organ metastasis, or renal vein thrombosis were not observed (Figures 2a-c). Radiological findings were compatible with low-grade RCC.

\section{Pathological Findings}

The patient underwent an open partial nephrectomy with pre-diagnosis of RCC based on radiological findings. The macroscopic examination revealed a partially wellcircumscribed, non-encapsulated, solid, and tan-brown lesion in $35 \times 32 \times 21 \mathrm{~cm}$ diameter. Central scar was not observed. The microscopic examination revealed tumor cells with oncocytic cytoplasm, irregular nucleus, nucleolus, and focal perinuclear halo in some areas in solid pattern (Figure 3a-b). Thus, differential diagnoses included oncocytoma, ChrRCC, low-grade oncocytic tumor (LOT), eosinophilic clear cell RCC, epithelioid hemangioendothelioma, succinate dehydrogenase-deficient RCC, and hybrid oncocytoma-chromophobe tumor. The immunohistochemical examination found a diffusely positive reaction for EMA, cytokeratin (CK) 7, and PAX8. A negative reaction for immunohistochemical cluster of differentiation (CD) 10, CD117, alpha methyacyl CoA racemase, RCC, HMB45, Melan A, and CK20 was recorded (Figure 4a-d). Therefore, the patient was diagnosed with LOT.

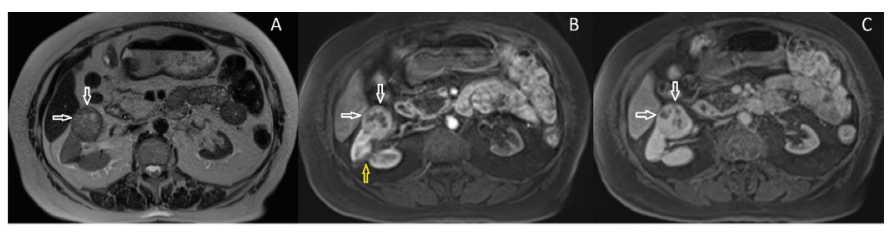

Figure 2. Dynamic enhanced MRI obtained in axial plan; T2W (a), arterial phase T1W (b), late phase T1W (c) images. The lesion is clearly seen in all images (white arrows). The mass without destruction is rapidly enhanced in the early arterial phase (b). Contrast washout is not observed in the late phase (c). Nonenhanced areas within the lesion are compatible with necrosis. The contour irregularity and notching seen in the right kidney middle section are compatible with the sequelae changes ( $b$, yellow arrow). Radiological findings were found compatible with renal cell carcinoma

MRI: Magnetic resonance imaging

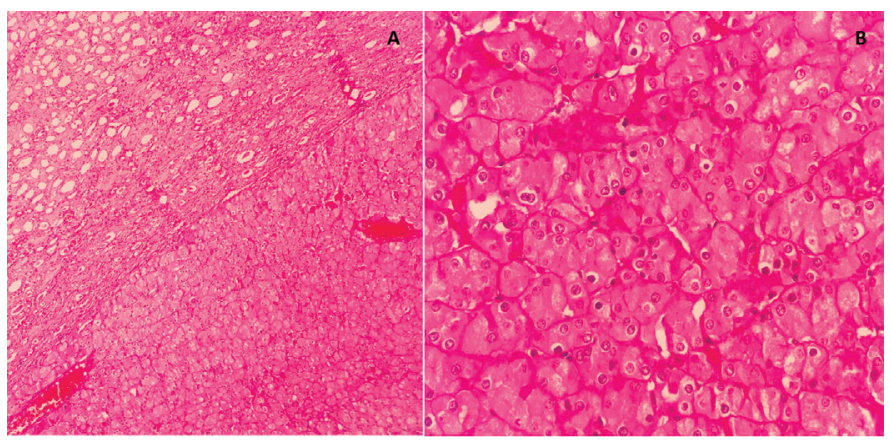

Figure 3. Microscopic features. Low-grade oncocytic tumor lacked a peripheral capsule and showed solid growth $(\mathrm{H \& E}, 40 \mathrm{X})(\mathrm{a})$, the cells demonstrated solid and compact acinar growth. The tumor cells had homogeneous oncocytic cytoplasm, uniformly round to oval nuclei (b) (H\&E, 400X)

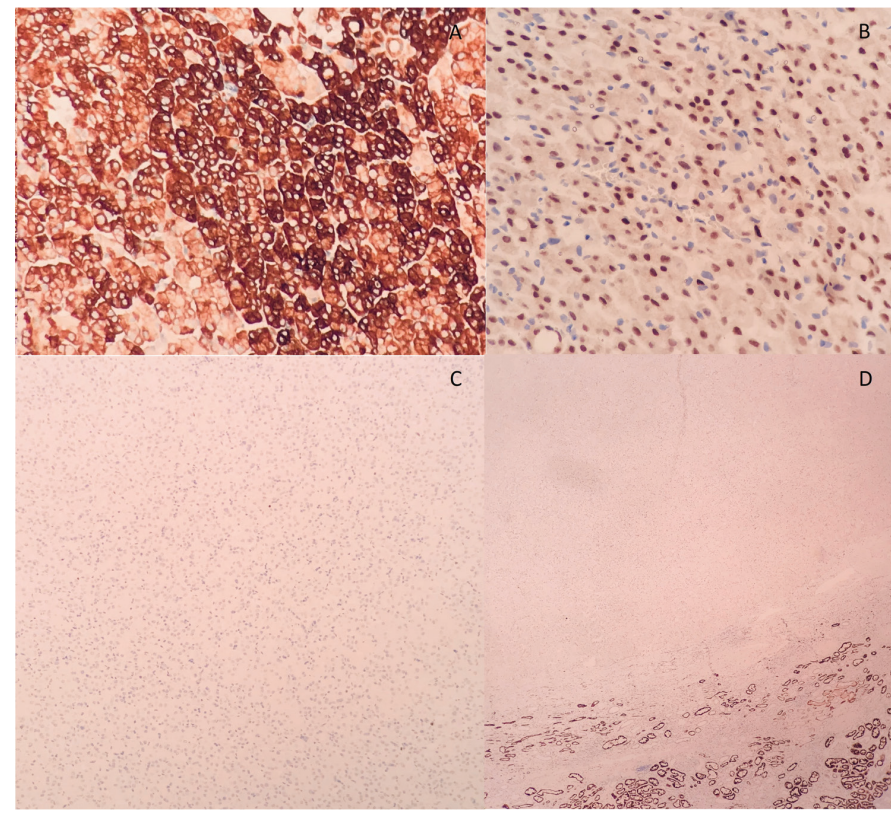

Figure 4. Immunohistochemical examination showing a positive reaction with CK7 (a) and PAX8 (b) and negative reaction with CD117 (c) and CD10 (d)

\section{Discussion}

Renal oncocytoma and ChrRCC were accepted as histological subtypes of renal tumors for years. ChrRCC is a malignant tumor, whereas renal oncocytoma is benign (4). A very little similarity was found between the classical histopathological appearance of ChrRCC and oncocytoma. However, serious problems are encountered in the eosinophilic variant differentiation of ChrRCC and oncocytoma. A great number of techniques, such as histochemical examinations, immunohistochemical examinations, chromosomal changes, molecular analyses, and electron microscopy were investigated to differentiate these two tumors for years, wherein immunohistochemical CD117 (KIT) and CK7 are most commonly used. CD117 and CK are positive in eosinophilic ChrRCC and oncocytoma. Moreover, CD117 and CK are positive in some oncocytomas with hybrid features. Using CD117 is helpful in differentiating typically CD117negative tumors. Prevalent CD117 positivity supports ChrRCC. CK7 is generally negative in oncocytoma or typically positive in $<5 \%$ of tumor cells (2). The oncocytic renal tumor differential diagnosis is presented in Table 1.

A study of 28 cases reported that LOTs showed a solid or compact nested growth pattern and the tumor cells had oncocytic cytoplasm with round to oval nuclei and focal perinuclear halo in microscopic examination. This study found CD117-/CK7+ in all cases. In addition, a negative reaction for CA9, CK20, vimentin, CK5/6, HMB45, Melan A, and CD15 was found. Frequent deletions detected at 19p13.3, 1p36.33, and $19 q 13.11$ on array comparative genomic hybridization and disomic status was recorded in 2 out of 9 cases (3). Moreover, "high-grade oncocytic tumors" showing different morphology and immunoprofile from LOTs are defined in literature (5).

In conclusion, LOTs are tumors with oncocytic morphology, CD117-/CK+ immunoprofile, lacking multiple chromosomal 


\begin{tabular}{|l|l|l|}
\hline \multicolumn{2}{|l|}{ Table 1. Differential diagnosis of low-grade oncocytic renal tumor } \\
\hline Diagnosis & Pathological findings & Immunohistochemistry \\
\hline Low-grade oncocytic tumor & $\begin{array}{l}\text {-Solid sheets and compact nests with gradual transition to trabecular areas } \\
\text {-Sharply delineated edematous stromal areas with loose cell growth }\end{array}$ & CD117-, CK7+, PAX8+ \\
\hline Chromophobe RCC, eosinophilic & $\begin{array}{l}\text {-Solid growth } \\
\text {-More prominent cell membranes, irregular (raisinoid) nuclei, perinuclear } \\
\text { halos }\end{array}$ & CD117+, CK7+, CD10- \\
\hline Oncocytoma & $\begin{array}{l}\text {-Tubulocystic growth } \\
\text {-Lacks perinuclear halos, central stromal "archipelaginous" areas are present }\end{array}$ & CD117+, CK7-/+ \\
\hline Clear cell RCC, eosinophilic & -At least focal clear cell areas, delicate vasculature in the background & CA9+, CD117-, CD10+ \\
\hline Epithelioid angiomyolipoma & -Epithelioid cells, pleomorphic, lacks perinuclear halos & $\begin{array}{l}\text { PAX8-, MelenA+, Desmin+, } \\
\text { HMBE45+, PANCK-, CK7- }\end{array}$ \\
\hline SDH- deficient RCC & $\begin{array}{l}\text {-Flocculent cytoplasm and vacuoles } \\
\text {-Lacks perinuclear halos }\end{array}$ & CD117-, SDH-, PANCK- \\
\hline RCC: Renal cell carcinoma & & \\
\hline
\end{tabular}

losses and gains, and exhibiting indolent clinical behavior. LOT shows oncocytic morphology in histopathological examination; however, its morphology does not completely fit into oncocytoma or eosinophilic ChrRCC. Therefore, the pathologist should support his diagnosis with immunochemistry. Estimating the actual LOT incidence is hard because these cases are previously reported as "eosinophilic ChrRCC," "oncocytic renal tumor/NOS," "unclassified/LOT," or "hybrid oncocytomachromophobe tumor." This study aimed to present LOT among newly defined oncocytic tumors to increase awareness.

\section{Acknowledgements}

Publication: The results of the study were not published in full or in part in form of abstracts.

Contribution: There is not any contributors who may not be listed as authors.

Conflict of Interest: No conflict of interest was declared by the authors.

Financial Disclosure: The authors declared that this study received no financial support.

\section{Ethics}

Informed Consent: Consent was obtained from the patient.
Peer-review: Externally peer-reviewed.

\section{Authorship Contributions}

Concept: Z.B., A.M.K., M.G., M.I.D., Design: Z.B., A.M.K., M.G., M.I.D., Data Collection or Processing: Z.B., A.M.K., M.G., M.I.D., Analysis or Interpretation: Z.B., A.M.K., M.G., M.I.D., Literature Search: Z.B., A.M.K., M.G., M.I.D., Writing: Z.B., A.M.K., M.G., M.I.D.

\section{References}

1. Perrino CM, Grignon DJ, Williamson SR, et al. Morphological spectrum of renal cell carcinoma, unclassified: an analysis of 136 cases. Histopathology 2018;72:305-319.

2. Williamson SR, Gadde R, Trpkov K, et al. Diagnostic criteria for oncocytic renal neoplasms: a survey of urologic pathologists. Hum Pathol 2017;63:149-156.

3. Trpkov K, Williamson SR, Gao Y, et al. Low-grade oncocytic tumour of kidney (CD117-negative, cytokeratin 7-positive): a distinct entity? Histopathology 2019;75:174-184.

4. Moch H, Cubilla AL, Humphrey PA, et al. The 2016 WHO classification of tumours of the urinary system and male genital organs-part a: renal, penile, and testicular tumours. Eur Urol 2016;70:93-105.

5. He H, Trpkov $\mathrm{K}$, Martinek $\mathrm{P}$, et al. "High-grade oncocytic renal tumor": morphologic, immunohistochemical, and molecular genetic study of 14 cases. Virchows Arch 2018;473:725-738. 